\title{
HIV Budding
}

National Cancer Institute

\section{Source}

National Cancer Institute. HIV Budding. NCI Thesaurus. Code C21244.

HIV Budding is a process in which newly generated viral RNA and protein complexes are assembled at, packaged in, and released from the cell surface membrane taking a piece of the cell membrane to form their own viral membranes. To bud from infected cells, HIV-1 employs several host proteins such as endosomal sorting protein Tsg 101, which functions in the Vps pathway. The L domain (PTAP) in the p6 domain of HIV-1 Gag interacts with the N-terminal domain of TSG101; the Vps28-binding domain of TSG101 inhibits budding. 